\title{
Efficacy in Reduction of Lung Lesions of a Toxin Expressing Whole-cell Vaccine Against Multiple Serovars of Actinobacillus Pleuropneumoniae in Growing Pigs
}

PREBEN MORTENSEN ( $\square$ preben.mortensen@ceva.com )

CEVA Animal Health: CEVA Sante Animale https://orcid.org/0000-0003-3059-8720

Nils Toft

IQinAbox ApS

Istvan Kiss

Ceva-Phylaxia Co. Ltd., SSIU

Vilmos Palya

Ceva-Phylaxia Co. Ltd., SSIU

Han Smits

Ceva-Phylaxia Co. Ltd., SID

Miklos Tenk

Ceva-Phylaxia Co. Ltd., Bio R\&D

\section{Research Article}

Keywords: Swine, Pleuropneumonia, Lung lesion score, Vaccine, Protection, Actinobacillus pleuropneumoniae, Coglapix

Posted Date: July 19th, 2021

DOI: https://doi.org/10.21203/rs.3.rs-719438/v1

License: (c) (i) This work is licensed under a Creative Commons Attribution 4.0 International License. Read Full License 


\section{Abstract}

Background: Actinobacillus pleuropneumoniae is a major economically significant bacterial respiratory pathogen of pigs, and vaccine use is considered an integral control method to prevent disease. The objective of this multi-study analysis was to evaluate the serovar independent efficacy in growing pigs of the C-vaccine (Coglapix ${ }^{\circledR}$, Ceva, France), which comprises whole cells of $A$. pleuropneumoniae serovars 1 and 2 expressing Apxl, Apxll and ApxIII toxins. Efficacy was based on protection against lung lesions, since there is good correlation between the severity/extension of lung lesions and losses induced by pleuropneumonia. Vaccine efficacy was determined against challenge with the most common serovars ( 1 , $2,4,5,6,7,9 / 11$ and 13) of $A$. pleuropneumoniae in a total of 13 studies of the same design and reproducibility was validated.

Results: Protection against homologous serovars 1 and 2 significantly reduced lung lesion scores (LLS) compared to the positive controls: $p=0.00007$ and $p=0.00124$, respectively. The protection against heterologous serovars $4,5,6,7,9 / 11$, and 13 also significantly reduced LLS: range $p=2.9 e-10$ to $p=$ 0.00953. Reproducibility between challenge studies was excellent with the estimated random effect of study (the fraction of the total variation attributed by differences between studies) being only $2.6 \%, 2.2 \%$ and $4.8 \%$ for three serovar 2 , two serovar $9 / 11$ and serovar 4 challenge studies, respectively. An outlier was the $35 \%$ of variation attributable to trial between the two serovar 6 challenges, possibly explained by a Streptococcus spp. outbreak.

Conclusions: A highly significant serovar independent reduction of pathological lung lesions by the Cvaccine was demonstrated for all the serovars tested $(1,2,4,5,6,7,9 / 11$ and 13). High levels of protection with similar significance-values were obtained for both homologous and heterologous serovar challenge. To our knowledge the aerosol chamber challenge concept based on accurate individual pig dosing and a standardized biologically weighted lung lesion scoring is the first to be validated statistically as reproducible and reliable. The $\mathrm{C}$-vaccine was demonstrated to be a good candidate to fulfil the demands in the field for an A. pleuropneumoniae-vaccine i.e., high protective capability against disease caused by multiple serovars.

\section{Background}

Actinobacillus pleuropneumoniae, the aetiological agent of swine pleuropneumonia, is responsible for substantial morbidity and mortality causing substantial economic losses in the global pork industry. Pleuropneumonia in its peracute and acute forms is mainly characterised by severely affected well-being and increased mortality. Pigs that survive infection, including after antimicrobial treatment, are likely to develop chronic disease characterised by reduced activity and appetite. Acute-peracute clinical signs are generally clear and distinct plus chronic pleurisy is easily diagnosed via slaughterhouse investigations ${ }^{1,2}$, 3,4. Pigs diagnosed with subacute pleuropneumonia show milder, less distinct clinical signs and lower fatality. While subclinical pleuropneumonia may involve pathological pneumonic lesions, no clinical signs may be apparent ${ }^{3,5}$. How accurate the distinction between the subclinical and subacute forms are in the 
individual case will rely highly on the frequency and quality of on-farm health monitoring. Some subclinical cases may very well be missed, for example, in farms where animals are co-infected with Mycoplasma hyopneumoniae. The subclinical form of $A$. pleuropneumoniae negatively affects growth rate and feed efficiency ${ }^{5}$ due to chronic lung lesions, like pleurisy and adherence together with a fibrinohaemorrhagic and necrotizing pleuropneumonia, as commonly seen at the abattoir ${ }^{6}$.

Estimations on the economic burden of this disease is mainly based on the occurrence of acute outbreaks characterized by high mortality and medical costs; few reports on production efficacy parameters like average daily weight gain (ADG) and/or feed conversion ratio (FCR), and even less have measured losses due to subacute and/or subclinical pleuropneumonia ${ }^{3}$. An analysis of five publications on 14 trials, found mean improvements due to antimicrobial intervention in ADG of $33.6 \%$ and FCR of $25.5 \%$, with high variations ${ }^{7}$. In a controlled long-term field study including a total of 33063 pigs, C-vaccinated (were compared to non-vaccinated for the control of acute pleuropneumonia, mortality was reduced by $28 \%$ ( $p=$ $0.011)$. However, the improvements on feed efficiency by $9.36 \mathrm{~kg}$ feed per pig produced $(p=0.023)$, and ADG by 40 per day or pen-efficacy by $6.3 \%(p<0.001)$ will mainly be attributable to subclinical-subacute pleuropneumonia occurring earlier than the observed recurrent outbreaks ${ }^{8}$.

The appearance of lung lesions, like pleurisy, at the abattoir, is often associated with A. pleuropneumoniae $(\mathrm{OR}=8.75)^{9,10}$, and can be linked to decreased $A D G$, increased FCR, prolonged stay in finishing compartment, and reduced carcass weight ${ }^{11,12}$. Pleurisy present at slaughter reduced lifetime weight gain by $1.25 \mathrm{~kg}$ on post trimming carcass weight, equal to $1.66 \mathrm{~kg}$ of live weight, in average. Also, a $10 \%$ increase of affected lung tissue was correlated of to a reduction of $3.3-4,6 \%$ on ADG ${ }^{13}$.

Lung lesion scoring is considered highly relevant for estimating severity and losses of respiratory disease, such as caused by $A$. pleuropneumoniae, at the farm level $14,15,16,17$. To investigate pleuropneumonia in all its possible manifestations, pathological evaluation of lung lesions appears to be the least biased method. Performing this evaluation close to pneumonic infection would seem to reveal the most accurate validation of the degree of pleuropneumonic impact on the individual pig.

Lung lesion scoring as the endpoint of measuring on A. pleuropneumoniae induced disease is widely accepted $18,19,2021,22,23,24,25,26,27,28,29,30$. A dose-response relation exists $31,18,22,23$. Depending on the challenge dose of the individual serovar one can achieve any stage of disease from absolute mortality 32 , $33,19,20,21,22$, even in bacterin vaccinated pigs ${ }^{21}$, to subacute ${ }^{18,24}$ even to subclinical pleuropneumonia $18,21,24$. However, due to high variation in disease inside $A$. pleuropneumoniae challenged groups $18,21,22$, $23,24,27,28,29,30,31$ a calculation of a dose-response will always produce a variation in lung lesions; least when mortality is the outcome. Here the lung lesions are severe and extensive affect $80-100 \%$ of the parenchyma $19,20,25,27,30,31,32,33$.

A. pleuropneumoniae is endemic world-wide and swine farms are often infected with more than one serovar. In some countries $80-90 \%$ of farms are estimated to be seropositive for $A$. pleuropneumoniae, with $1-6$ out of 7 serogroups present at the same time: $93 \%$ having $>=2$ and $80 \%$ having $>=3$ 
simultaneous Ap-serogroups present ${ }^{34,35}$. The prevalence of serovars varies between countries, regions of countries, and by year of investigation $35,36,37,38,39,40$.

So far nineteen A. pleuropneumoniae serovars have been classified worldwide ${ }^{41}$. However, as the difference between serovar 9 and 11 is only one amino acid in the complete CPS loci and they have identical toxin profiles (Apxl, ApxII), these two serovars can be considered as one: serovar 9/11 ${ }^{42}$. Strains belonging to different serovars are highly different in virulence and in some cases different strains of the same serovars can express different pathogenicity features; usually due to different Apx toxin profiles investigation $35,36,37,38,43$. More extrinsic factors like general stress $3,43,44$, poor air quality and climatic control, particularly high ambient temperature variations over the day, are associated with increased severity of pathological lesions caused by A. pleuropneumoniae, even in the case of what are considered low virulent $A$. pleuropneumoniae strains ${ }^{3,6}$.

A. pleuropneumoniae has several virulence factors, some are well described, and several are under investigation. The three exotoxins: ApxI-III and lipopolysaccharide (LPS) have been proven responsible for lung lesions, but at the same time are both immunogenic and can induce protective immunity ${ }^{1,3,46}$. Many A. pleuropneumoniae virulence factors have been described ${ }^{46}$ including outer membrane proteins (OMPs), some of which are immunogenic and therefore potential vaccine candidates ${ }^{47}$.

The variation in virulence between A. pleuropneumoniae serovars is mainly determined by the production of one or two of the ApxI-III toxins. These exotoxins providing nutrients for further growth and activity via lysis of the nearby cells in the lung tissue including neutrophils and macrophages ${ }^{48,49}$. LPS are both adhesion factors, allowing for colonisation and the production of exotoxins, and at the same time enhancing the cytotoxic effects of ApxI-III $50,51,52,53$.

Several commercial vaccines are available which differ in their composition and can be appointed into one of three $A$. pleuropneumoniae vaccine categories: 1) killed $A$. pleuropneumoniae whole-cell components only (bacterins); 2 ) subunit vaccines containing ApxI-III toxins only; and 3) a combination of these ${ }^{47}$. With distinct differences in efficiency, they all reduce clinical signs, but none can fully prevent infection and colonization ${ }^{54}$. Antibodies against ApxI-III are responsible for the serovar-independent protection against lung lesions $3,6,46,47$. Due to limited cross protection between the serovars, bacterin vaccines lack efficacy compared to ApxI-III combined bacterin vaccines; pure toxoids vaccines lack in general protective capacity due to lack of LPS and other cell wall components $55,56,57,58$. A pleuropneumoniae vaccine group 3 above is quite heterogenous, with a wide variety in composition, from a subunit vaccine containing the ApxI-III and only one of the cell wall OMPs, to the vaccine evaluated in this study based on whole-cell components of two serovars, together expressing all three of the ApxI-III toxins, and to vaccines containing whole-cell components of several A. pleuropneumoniae serovars together with some exotoxins.

A combination of the three exotoxins, ApxI-III with LPS, and likely more of the abundant cell-wall based antigens 56,58 , induces a strong and specific cell mediated immune response that can confer serovar 
independent protection ${ }^{3}$. This is an effective design for an efficacious serovar-independent vaccine, feasible for $A$. pleuropneumoniae prophylaxis to: increase animal well-being, reduce antimicrobial use, and reduce losses due to pleuropneumonia in all its manifestations at any A. pleuropneumoniae-endemic farm at any time $\mathrm{e}^{3,6,47}$.

The objective of this study was to evaluate the efficacy of a vaccine comprising whole cells of $A$. pleuropneumoniae serovars 1 and 2 which in combination express Apxl, ApxII and ApxIII to protect against pleuropneumonic lungs lesions following challenge with multiple prominent serovars of $A$. pleuropneumoniae in growing pigs.

\section{Materials \& Methods}

Data from thirteen studies each including one of the eight $A$. pleuropneumoniae serovars $1,2,4,5,6,7$, 9/11 and 13 performed over the period of 2011 to 2020 were available (Table 1). Where data on multiple studies with the same serovar were available weighted lung lesion score (LLS) of the vaccinated group of pigs (Vac) versus the non-vaccinated pigs in the positive control group (Pos) were pooled and analysed while taking the potential effect of study into account (Table 1). Also, variance between studies on the same serovars were analysed to estimate quality of repeatability. 
Table 1

List of $A$. pleuropneumoniae challenge trials used in in this analysis. The two studies performed by Ceva R\&D are intranasal dosed challenges (IN). the 11 studies performed by Ceva SSIU are all aerosol chamber dosed challenges.

\begin{tabular}{|c|c|c|c|c|c|c|c|c|}
\hline Serovar & Origin & Strain ID & $\begin{array}{l}\text { Dose } \\
\text { CFU/pig }\end{array}$ & $\begin{array}{l}\text { Test } \\
\text { Group }\end{array}$ & $\begin{array}{l}\text { Pigs } \\
\text { / group }\end{array}$ & $\begin{array}{l}\text { Year } \\
\text { of } \\
\text { study }\end{array}$ & $\begin{array}{l}\text { Pig } \\
\text { breed }\end{array}$ & $\begin{array}{l}\text { Official } \\
\text { approval ID }\end{array}$ \\
\hline \multirow[t]{3}{*}{1} & \multirow[t]{3}{*}{ Denmark } & \multirow{3}{*}{$\begin{array}{l}\text { App. St1 ch } \\
\text { BS5689 }\end{array}$} & \multirow{3}{*}{$\begin{array}{l}4 \times 10^{8} \\
\text { IN }\end{array}$} & Vac & 8 & \multirow[t]{3}{*}{2012} & \multirow{3}{*}{$\begin{array}{l}\text { Hungaro- } \\
\text { Seghers }\end{array}$} & \multirow{3}{*}{$\begin{array}{l}\text { BA01/2005- } \\
1 / 2010\end{array}$} \\
\hline & & & & Vac & 8 & & & \\
\hline & & & & Pos & 7 & & & \\
\hline \multirow[t]{6}{*}{2} & \multirow[t]{6}{*}{ Hungary } & \multirow{6}{*}{$\begin{array}{l}\text { App. St2 ch } \\
2008 / 3+2\end{array}$} & \multirow[t]{2}{*}{$1 \times 10^{6}$} & Vac & 11 & \multirow[t]{2}{*}{2015} & \multirow{2}{*}{$\begin{array}{l}\text { Topigs- } \\
\text { Norsvin } \\
\text { x PIC }\end{array}$} & \multirow{2}{*}{$\begin{array}{l}\text { BA01/2005 } \\
\text { és 1/2010- } \\
1 / 2012\end{array}$} \\
\hline & & & & Pos & 11 & & & \\
\hline & & & \multirow[t]{2}{*}{$1 \times 10^{7}$} & Vac & 9 & \multirow[t]{2}{*}{2017} & \multirow[t]{2}{*}{ Danbred } & \multirow{2}{*}{$\begin{array}{l}\text { BAO2/2000- } \\
43 / 2017\end{array}$} \\
\hline & & & & Pos & 10 & & & \\
\hline & & & \multirow[t]{2}{*}{$1 \times 10^{8}$} & Vac & 10 & \multirow[t]{2}{*}{2014} & \multirow{2}{*}{$\begin{array}{l}\text { Hungaro- } \\
\text { Seghers }\end{array}$} & \multirow{2}{*}{$\begin{array}{l}\text { BA01/2005 } \\
\text { és 1/2010- } \\
1 / 2012\end{array}$} \\
\hline & & & & Pos & 10 & & & \\
\hline \multirow[t]{4}{*}{4} & \multirow[t]{4}{*}{ Spain } & \multirow[t]{4}{*}{ App.90993 } & \multirow[t]{2}{*}{$1 \times 10^{8}$} & Vac & 10 & \multirow[t]{2}{*}{2018} & \multirow[t]{2}{*}{ Danbred } & \multirow{2}{*}{$\begin{array}{l}B A 02 / 2000 \\
43 / 2017\end{array}$} \\
\hline & & & & Pos & 9 & & & \\
\hline & & & $1 \times 10^{8}$ & Vac & 20 & 2020 & Danbred & $\begin{array}{l}\text { BAO2/2000- } \\
43 / 2017\end{array}$ \\
\hline & & & & Pos & 20 & & & \\
\hline
\end{tabular}

\begin{tabular}{|c|c|c|c|c|c|c|c|c|}
\hline \multirow[t]{2}{*}{5} & \multirow[t]{2}{*}{ Italy } & \multirow{2}{*}{$\begin{array}{l}\text { App St. } 5 \\
\text { 13/TA }\end{array}$} & \multirow{2}{*}{$1 \times 10^{6}$} & Vac & 10 & \multirow[t]{2}{*}{2011} & \multirow{2}{*}{$\begin{array}{l}\text { Hungaro- } \\
\text { Seghers }\end{array}$} & \multirow{2}{*}{$\begin{array}{l}\text { BA01/2005- } \\
1 / 2010\end{array}$} \\
\hline & & & & Pos & 10 & & & \\
\hline \multirow[t]{4}{*}{6} & \multirow[t]{4}{*}{ Denmark } & \multirow{4}{*}{$\begin{array}{l}\text { App. J.no. } \\
101059+ \\
2 S P\end{array}$} & \multirow{2}{*}{$1 \times 10^{8}$} & Vac & 10 & \multirow[t]{2}{*}{2018} & \multirow[t]{2}{*}{ Danbred } & \multirow{2}{*}{$\begin{array}{l}\text { BAO2/2000- } \\
43 / 2017\end{array}$} \\
\hline & & & & Pos & 9 & & & \\
\hline & & & \multirow[t]{2}{*}{$1 \times 10^{8}$} & Vac & 18 & \multirow[t]{2}{*}{2019} & \multirow[t]{2}{*}{ Danbred } & \multirow{2}{*}{$\begin{array}{l}\text { BAO2/2000- } \\
43 / 2017\end{array}$} \\
\hline & & & & Pos & 14 & & & \\
\hline \multirow[t]{2}{*}{7} & \multirow[t]{2}{*}{ Hungary } & \multirow{2}{*}{$\begin{array}{l}\text { App. St7 ch } \\
\text { CH.G-I/7- } \\
7 / 12\end{array}$} & \multirow{2}{*}{$\begin{array}{l}2.8 \mathrm{X} \\
10^{8} \mathrm{IN}\end{array}$} & Vac & 18 & \multirow[t]{2}{*}{2012} & \multirow{2}{*}{$\begin{array}{l}\text { Hungaro- } \\
\text { Seghers }\end{array}$} & \multirow{2}{*}{$\begin{array}{l}\text { BA01/2005- } \\
1 / 2010\end{array}$} \\
\hline & & & & Pos & 14 & & & \\
\hline \multirow[t]{2}{*}{ 9/11 } & \multirow[t]{2}{*}{ Hungary } & \multirow{2}{*}{$\begin{array}{l}\text { App. St.9 } \\
\text { ch (B- } \\
2011)\end{array}$} & \multirow[t]{2}{*}{$1 \times 10^{6}$} & Vac & 11 & \multirow[t]{2}{*}{2012} & \multirow{2}{*}{$\begin{array}{l}\text { Hungaro- } \\
\text { Seghers }\end{array}$} & \multirow{2}{*}{$\begin{array}{l}\text { BA01/2005 } \\
1 / 2010\end{array}$} \\
\hline & & & & Vac & 10 & & & \\
\hline
\end{tabular}




\begin{tabular}{|c|c|c|c|c|c|c|c|c|}
\hline Serovar & Origin & Strain ID & $\begin{array}{l}\text { Dose } \\
\text { CFU/pig }\end{array}$ & $\begin{array}{l}\text { Test } \\
\text { Group }\end{array}$ & $\begin{array}{l}\text { Pigs } \\
\text { / group }\end{array}$ & $\begin{array}{l}\text { Year } \\
\text { of } \\
\text { study }\end{array}$ & $\begin{array}{l}\text { Pig } \\
\text { breed }\end{array}$ & $\begin{array}{l}\text { Official } \\
\text { approval ID }\end{array}$ \\
\hline & & & & Pos & 10 & & & \\
\hline & & & \multirow[t]{2}{*}{$1 \times 10^{8}$} & Vac & 10 & \multirow[t]{2}{*}{2014} & Hungaro- & BA01/2005 \\
\hline & & & & Pos & 10 & & Seghers & $\begin{array}{l}\text { és 1/2010- } \\
1 / 2012\end{array}$ \\
\hline \multirow[t]{2}{*}{13} & \multirow[t]{2}{*}{ Spain } & \multirow{2}{*}{$\begin{array}{l}\text { App.99865 } \\
+1\end{array}$} & \multirow[t]{2}{*}{$1 \times 10^{7}$} & Vac & 20 & \multirow[t]{2}{*}{2020} & \multirow[t]{2}{*}{ Danbred } & \multirow{2}{*}{$\begin{array}{l}\text { BA02/2000- } \\
43 / 2017\end{array}$} \\
\hline & & & & Pos & 20 & & & \\
\hline
\end{tabular}

The trial designs were all the same (see Trial design) and in accordance with the European Pharmacopeia 59. All trials were performed by Ceva Research and Development (R\&D) Department or Ceva Scientific Support and Innovation Unit (SSIU) in Hungary.

\section{The vaccine}

The vaccine tested was Coglapix ${ }^{\circledR}$ (Ceva Santé Animale, France) hereafter referred to as C-vaccine. The Cvaccine is based on whole cells of $A$. pleuropneumoniae serovars 1 and 2 expressing Apxl, ApxII and ApxIII. Over the span of years, the vaccine composition and quality control has not changed. Apart from the cross-protective Apx-toxins, this vaccine contains all principal cell wall structures of $A$. pleuropneumoniae bacteria in undetermined quantities which contribute to A. pleuropneumoniaeprotective immune response: LPS, OMP's and the several other cell wall components.

\section{Serotyping of the challenge strains}

Strains belonging to different serovars were isolated from clinical cases of swine pleuropneumonia and serotyped using hyper immune sera by indirect haemagglutination as described previously 60 .

Serotyping of all A. pleuropneumoniae strains was confirmed in a multiplex-PCR based on capsular loci carried out as described by Bossé and colleagues ${ }^{61}$.

\section{Calibration and preparation of the challenge strains}

Strains were assessed for their ability of growth in liquid culture in a Tryptic soy broth supplemented with yeast extract and nicotinamide adenine dinucleotide solution in shake-flasks rotated at $180 \mathrm{rpm}$ and kept at $37^{\circ} \mathrm{C}$. Their growth curve was analysed using sampling at pre-determined sampling points and subsequent optical density (OD) measuring at different wavelengths using a standard laboratory photometer. At each sampling point, the cultures were subjected to colony forming unit (CFU) counts using standard bacteriological techniques. The OD and CFU values were then aligned and the strainspecific, optimal wavelengths were determined. After this initial procedure, in each case when a challenge 
trial was performed, the strain used was prepared in shake flasks under regular OD monitoring and stopped when reaching the desired live titre based on the OD-CFU calibration curve.

\section{Aerosol dosing technique}

Challenge strains were propagated and used for the test when $10^{9} \mathrm{CFU} / \mathrm{ml}$ concentration was reached. The $A$. pleuropneumoniae stock was diluted in sterile PBS to achieve the optimal required $10^{6}, 10^{7}$ or $10^{8}$ CFU/animal treatment dosage as shown in Table 1. Actual calculations were made at the test site, using the following parameters to introduce 1 dose/animal during the aerosol treatment to the chamber:

- Pig body weight 62 and volume

- Number of pigs placed in the chamber for one run $(6,8$ or 10$)$

- Volume of chamber.

- Volume of liquid, turned to aerosol by the ultrasonic nebulizer in 10 minutes (usually 100-150 ml, depending on air temperature and humidity)

The pigs were evenly distributed and secured in the chamber by partition fences; aerosol was created by an ultrasonic humidifier and uniformly dispersed by internal ventilation. After 10 minutes of treatment, the pigs were kept in the chamber for an additional 2 minutes with the nebulizer switched off, to allow complete uptake of the aerosol droplets (fresh air was provided during this time to allow normal breathing). Before the first run, the chamber was moisturized with the nebulizer to prevent aerosol loss caused by adherence to the dry surfaces; before each run piglets introduced to the chamber are given a couple of minutes of ease to ensure normal respiration before the doors are closed and the challenge is initiated. This aerosol chamber concept is developed by Palya and Kiss, and inspired by previous work on aerosol chambers ${ }^{18,32,63}$.

\section{Intranasal (IN) challenge}

Production of the challenge strain and calibration of the challenge dose was as described above. The cultures were prepared in $10 \times$ concentration of the desired challenge titre and diluted in sterile PBS to reach the working concentration. Each animal received $5 \mathrm{ml}$ of challenge dose into each nostril using intranasal cannulas; the exact individual animal dose is shown in Table 1.

\section{Pre-trial determination of challenge dose}

Prior to using the strains in vaccine challenge trials, challenge dose calibration studies were performed. In these trials, three groups of 10 non-vaccinated $A$. pleuropneumoniaenegative pigs were challenged with doses of $10^{6}, 10^{7}$ or $10^{8}$ CFUs, monitored daily for clinical signs and euthanized one week later. Mortality and LLS were evaluated to select the optimal challenge dose to be used: a mortality of $20-30 \%$ in the non-vaccinated control group.

For A. pleuropneumoniae 2 and $A$. pleuropneumoniae 9/11 the pleuropneumonic impact of different concentrations of challenge dose were investigated via LLS to evaluate the $A$. pleuropneumoniae 
protective capabilities of the vaccine for different challenge loads.

\section{Inclusion criteria}

Pigs of either sex and of different breeds changing over time (Table 1) were recruited from farms free of $A$. pleuropneumoniae, Mycoplasma hyopneumoniae, toxin-positive Pasteurella multocida (progressive atrophic rhinitis), porcine reproductive and respiratory syndrome virus, Aujeszky's disease virus, classical swine fever virus and African swine fever virus based on regular PCR and/or serology tests performed either by government or private labs. Also, the animals had no previous clinical history of infection by Streptococcus suis and Glaesserella parasuis.

Any animal selected for inclusion in A. pleuropneumoniae challenge trials had to be negative in the ApxIV ELISA (IDEXX APP-ApxIV Ab) test when serum sampled at 5-6 weeks of age, confirming that they were negative for both A. pleuropneumoniae infection and colostral A. pleuropneumoniae antibodies.

\section{Trial design}

The challenge trials were performed using the same overall study design. The only difference was the use of an aerosol chamber challenge model by SSIU and an intra-nasal (IN) application by R\&D (Table 1). Detailed methods are given below.

Pigs at the age of 7-8 weeks, were randomly assigned to either non-vaccinated or vaccinated groups and housed indoors, with controlled temperature and ventilation. Each pig of the vaccine group (Vac) received the first $2 \mathrm{ml}$ dose of the C-vaccine by intramuscular injection (D0). Three weeks later, D21, the pigs of the Vac group received a second $2 \mathrm{ml}$ dose intramuscular of the same vaccine; the controls received no treatment. Pigs were randomised according to bodyweight and staff responsible for the daily care and monitoring of the pigs were not involved in vaccination and unaware of which pigs belonged to which test-groups.

At D42 all pigs individually received pre-determined equal doses of the relevant virulent $A$. pleuropneumoniae strains either by application in an aerosol chamber, or by the IN route, as described above.

At D49, one-week post-challenge, the trials were terminated. All live pigs were humanely euthanized and pathoanatomically evaluated to establish the individual lung lobe lesions to calculate the individual LLS. Persons performing the pathoanatomical evaluation were not involved in vaccination and unaware of which pigs belonged to which test-groups.

For A. pleuropneumoniae 2, three studies, for $A$. pleuropneumoniae 4, 6 and 9/11, two studies, and for the remaining $A$. pleuropneumoniae serovars, one study were included in the analyses.

\section{Post-mortem evaluation of weighted lung lesion score (LLS)}

In the vaccination-challenge trials, all animals euthanised on day 7 post-challenge, D49, were subjected to necropsy to investigate the pathological changes due to actinobacillosis. Evaluation of the post-mortem 
lesions in the lungs and on the pleura were performed blind and in accordance with a previously described scoring system ${ }^{15}$. All seven lobes of the lung of each pig in trial were examined and each lobe scored on prevalence of pathological lesions of pneumonia and/or pleuritis (pleuropneumonia). Score valuing was according to the size of the affected area: absence $=$ score $0,1-20 \%=$ score $1,21-40 \%=$ score $2,41-60 \%$ $=$ score $3,61-80 \%=$ score 4 , and $81-100 \%=$ score $5^{15}$.

Weighting factors were applied on all individual lung-lobe scorings according to the relative size of each lobe in the lung of each pig: right-cranial $=0.07$, right-middle $=0.15$, right-caudal $=0.35$, accessory-lobe $=$ 0.05 , left-cranial $=0.04$, left-middle $=0.09$, and left caudal $=0.25{ }^{63}$. Pigs that had died during the week following challenge and before termination of the trial were given the maximum lung lesion score of 5 . This way each pig lung ended up with a total LLS of $0-5$; the more lesions the higher the score.

\section{Statistical analyses}

The effect of vaccine on LLS was analysed using linear (mixed) models separately for each $A$. pleuropneumoniae serovar. If more than one study was available, a random effect of study to account for the possible clustering of effects within a study was included. To assess the importance of the between study variation, the intraclass correlation coefficient (ICC) was calculated as the proportion of the total variation attributed to the random effect. For the outcome (LLS), a limit of detection (LOD) was defined as half the minimum observed LLS. The LOD was added to all LLS before it was log transformed to improve the underlying assumption about normal distribution of data. All analyses were done in $\mathrm{R}^{65}$, using the Ime $4{ }^{66}$ package for statistical analyses of mixed effects models with the ImerTest ${ }^{67}$ package for testing of significant effects.

\section{Results}

The protection of the $\mathrm{C}$-vaccine against the homologous $A$. pleuropneumoniae serovars 1 and 2 strains was demonstrated with highly significant reductions of LLS compared to the positive controls: $p=$ 0.00007 and $p=0.00124$ respectively (Table 2 ). The protection of the $\mathrm{C}$-vaccine against the heterologous A. pleuropneumoniae serovars $4,5,6,7,9 / 11$, and 13 was demonstrated with equally highly significant reductions in LLS: $p=2.9 \mathrm{e}-10$ to $p=0.00953$ (Table 2). The negative controls in all studies stayed $A$. pleuropneumoniae negative and without any LLS to be observed. 
Table 2

Sample size, mean Lung Lesion Score (LLS) and standard deviation (SD) for vaccine groups and positive controls for $A$. pleuropneumoniae serovars (A.p.) $1,2,4,5,6,7$, and $9 / 11$. The $p$-value is for the test of a difference between vaccine and positive controls within each $A$. pleuropneumoniae type. Significance is considered when the $p$ value $<0.05$

\begin{tabular}{|llllllll|}
\hline \multicolumn{7}{|l}{ Vaccine } & \multicolumn{5}{l|}{ Positive Control } \\
\hline A.P. serovar & $\mathrm{n}$ & LLS & SD(LLS) & $\mathrm{n}$ & LLS & SD(LLS) & P-value $^{1}$ \\
\hline A.p. 1 & 16 & 0.23 & 0.31 & 7 & 1.96 & 1.14 & 0.00007 \\
\hline A.p. 2 & 30 & 0.75 & 1.22 & 31 & 2.11 & 2.05 & 0.00124 \\
\hline A.p. 4 & 30 & 0.65 & 0.41 & 29 & 1.46 & 1.25 & 0.00044 \\
\hline A.p. 5 & 10 & 0.18 & 0.54 & 10 & 1.18 & 1.61 & 0.00953 \\
\hline A.p. 6 & 28 & 0.71 & 0.60 & 23 & 1.56 & 1.13 & 0.00195 \\
\hline A.p. 7 & 17 & 0.04 & 0.10 & 17 & 1.17 & 1.15 & $2.9 \mathrm{e}-10$ \\
\hline A.p. 9/11 & 31 & 2.26 & 1.89 & 20 & 3.84 & 1.82 & 0.00663 \\
A.p. 13 & 20 & 1.03 & 1.44 & 20 & 2.66 & 1.93 & 0.00319 \\
\hline
\end{tabular}

${ }^{1}$ All tests of significance were done on the log(LLS) to improve the underlying assumptions of the analysis

Some variation in mean LLS was observed between A. pleuropneumoniae serovars, supporting the decision to analyse each A. pleuropneumoniae serovar separately (Table 2). For A. pleuropneumoniae 2, the estimated random effect of study was (variance $=0.071$ ) and the residual variance was 1.860 , which implies an ICC $=0.05 /(0.05+1.88)=0.026$, i.e., only $2.6 \%$ of the total variation was due to differences between studies. For $A$. pleuropneumoniae $9 / 11$ the ICC $=0.02 /(0.02+0.87)=0.022$, i.e., $2.2 \%$, and for $A$. pleuropneumoniae 4 ICC $=0.05 /(0.052+0.99)=0.048$, i.e., $4.8 \%$. This suggests a standardized set-up, where the effect of study essentially can be ignored in the analyses. However, for A. pleuropneumoniae 6, the ICC $=0.54 /(0.54+1.01)=0.35$, i.e., $35 \%$, suggesting that there were marked differences between these two studies.

\section{Discussion}

The concept of combining the Apxl, ApxII and ApxIII 3, 6, 46,47 with cellular components of $A$. pleuropneumoniae ${ }^{55,57}$ has been demonstrated to result in a highly effective serovar-independent vaccine that reduces lung lesions and mortality, and improves production performance. The use of such an efficacious vaccine will increase animal well-being, and reduce both antimicrobial use and economic losses due to endemic pleuropneumonia ${ }^{3,6,47}$. 
To our knowledge, this is the most exhaustive testing on any $A$. pleuropneumoniae-vaccine, whether approved to be serovar independent or not by the relevant authorities. In these studies, we have analysed the efficacy of the $\mathrm{C}$-vaccine in protecting against lung lesions of eight different virulent field strains of $A$. pleuropneumoniae $(1,2,4,5,6,7,9 / 11$ and 13$)$; six heterologous and two homologous with the serovars on which the vaccine is based. We found a significant reduction in LLS for the vaccinated groups compared to the positive controls. This implies that the vaccine is capable of inducing serovarindependent protection and is a valuable characteristic for optimizing the control of $A$. pleuropneumoniae related pig health problems.

The majority of the studies we carried out used an aerosol chamber. When considering challenge models, for most investigators, the choice is between IN or aerosol chamber challenge (AC). IN has an inherited accuracy in applied dose but is labour intensive and comparatively more expensive. In addition, dependent on pig handling and dose application (e.g., sedation/non-sedation), IN is potentially more stressful which can increase respiratory rate, hence respiratory volume and can affect the planned dose. With AC, skilful pig handling can ensure acceptance of the animals to the chamber and less stress. Our results indicate that reproducible protection studies can be performed using aerosol chambers with, to our knowledge, the Ceva Phylaxia aerosol chamber $A$. pleuropneumoniae challenge concept, being the only one validated for reproducibility using the intraclass correlation coefficient (ICC). Where determined, reproducibility between challenge studies was high, hence the outcome-of-trial data can be considered of high reliability, reflecting accurate individual challenge dose calculations, and basing the lung lesion scoring on standardized methodology ${ }^{15}$ adapted to the biological appearance of the lung ${ }^{64}$ achieving standardized, reproducible, weighted lung lesion score (LLS).

The variations attributable to differences between studies are very low: $2.6 \%, 2.2 \%$ and $4.8 \%$ for three $A$. pleuropneumoniae 2, two A. pleuropneumoniae 9/11 and two A. pleuropneumoniae 4 challenge studies, respectively. An outlier is the $35 \%$ of variation attributable to trial between the two A. pleuropneumoniae 6 challenges. An explanation could be that pleuritis was generally observed in a larger proportion of the animals in the 2019-study. Bacteriology demonstrated the presence of Streptococcus spp. in these samples. Significant improvement in LLS compared to the control group was still observed in this trial alone, and even more so when analysed together with the 2018-study. That infection with other pathogens, e.g., Bordetella pertussis, can affect lesion score in A. pleuropneumoniae challenged animals has been documented by others ${ }^{30}$.

Others have used aerosol chambers to challenge pigs with $A$. pleuropneumoniae $18,19,20,21,22,23,24,25,26$, $27,28,29,30,31,32,33$. In these sixteen publications five common and important serovars were investigated: seven on serovar $1^{19}, 20,21,22,31,32,33$, four on serovar $7^{26,28,29,30}$, two on serovar $2^{23,24}$, one on serovar 5 only 25 , one on serovars 2,5 and $6^{18}$, and one on serovars 2 and $9^{27}$. Serovars 2,5 and 6 were concluded as being of moderate to high virulence ${ }^{18}$, but this was based on small numbers of animals being investigated, and the result with serovar 5 can be considered surprising given that this is normally considered as of high virulence ${ }^{48}$. Serovar 7 was considered as moderately virulent ${ }^{30}$. Based on very high doses in identical trial designs, serovars 1 and 5 appear comparable in virulence measured on mortality 
only 20,25 . When comparing dosage and outcome empirically across the heterogenous trial designs, serovar 1 stands out as the most virulent closely followed by serovars 5 and 9, placing serovars 2, 6 and 7 as moderate to highly virulent. However, most of these studies, like ours, were not designed to reveal differences in virulence, rather dosing was aimed at obtaining similar disease severity distributions in the positive control groups to enable assessment of vaccine protection. Nonetheless, our data indicates broad agreement with the literature in that serovars 1,5,9/11 are the most virulent, serovars 2 and 13 of slightly lesser virulence, and serovars 4, 6 and 7 as moderate-to-highly virulent. It should be noted that the serovar 2 isolate we used was from Europe which expresses ApxII and ApxIII which is of higher virulence than serovars 2 isolates from North America which typically only express ApxII ${ }^{37}$. A definite rating of the virulence of different serovars by AC would require fully standardised extensive head-to-head trials to be carried out in a reproducible challenge model similar to that presented and validated in this publication.

In this study we have used the LLS to assess vaccine efficacy after aerosol challenge to $A$. pleuropneumoniae. In the 16 aerosol challenge papers previously reported, 28 tests groups can be identified, with five reporting mortality and describing lung lesions in general pathological terms $19,31,32$, 33 , three are using in-house models including other than the lungs $18,21,22$, two calculate percentage of lung tissue affected ${ }^{19,25}$. Only seven score lung lesions with the standard scheme of Hannan and colleagues $15,23,24,26,27,28,29,30$. None of them used a weighted lung lesion score which takes into account the size of the individual lung lobes for optimal comparison between pigs and groups.

Also, the days from challenge to scoring varies substantially between the 28 groups: twelve are in the interval of 15 to 22 days and only focused on chronic lesions $19,21,22,23,26,27,28,29,30,33$, three are intermediate from 12-14 days ${ }^{19,33}$, nine are focused on acute, subacute and subclinical lesions in the interval of $5-7$ days $19,20,23,24,25,27,29,31,32$, and one was assessed only at 24 hours ${ }^{19}$; in three publications comparisons were made between groups where dead animals were not part of the analyses are not included here $22,27,29$. Finally, numbers are predominantly small with only $5 / 28$ groups using 10 animals or above $21,22,30$, another five used 8 pigs $19,23,25,26,28$, and the majority using less pigs in a test group $18,19,20,22,23,24,27,29,30,31,32,33$. Thus, the variation in both dosing and assessment methodology severely complicates comparisons with other reported aerosol challenge studies. Further comparative studies would best be undertaken in a highly standardised model with reproducible methodology, such as that we report here. Also, further research in methods to validate $A$. pleuropneumoniae induced pleuropneumonic losses in general is key, but of particular interest for further evaluation of the subclinical/subacute forms ${ }^{3,18,21,24}$. In a world of reducing antimicrobial use, the ability to perform exact cost-benefit analyses on different $A$. pleuropneumoniae control strategies are already of great importance, and involvement of systematic LLS is likely to be an integral component of such schemes ${ }^{14}$.

\section{Conclusions}

The C-vaccine was highly effective, providing serovar-independent highly significant reductions of LLS in multiple challenges with different $A$. pleuropneumoniae serovars 1, 2, 4, 5, 6, 7, 9/11 and 13 . 
To our knowledge the aerosol chamber challenge concept is the only one validated for reproducibility, basing challenge on individual pig challenge doses and weighted lung lesions scores for the most accurate biologically evaluation of disease and vaccine protection against $A$. pleuropneumoniae disease.

\section{Declarations}

\section{Ethics approval and consent to participate}

The trial designs were all the same and in accordance with the European Pharmacopeia ${ }^{59}$. All trials were performed by Ceva Research and Development (R\&D) Department or Ceva Scientific Support and Innovation Unit (SSIU) in Hungary.

All studies followed local law and regulations. Authorization was provided by the Government Office of Baranya County Food Chain-Safety and Animal Health Department, Hungary. Individual study approval ID noted (Table 1).

The studies were conducted in accordance with the provision Directive 2010/63/EU (still in force), Hungarian act XXVII/1998 (still in force, with regular updates in content) and the Hungarian Ministerial Decree No. 243/1998, replaced the 15.04.2013 by the 40/2013. (II. 14.) Hungarian Governmental Decree, prepared according to the Directive 2010/63/EU.

\section{Consent for publication}

Not applicable

\section{Availability of data and materials}

The datasets used and/or analysed during the current study are available from the corresponding author on reasonable request.

\section{Competing interests}

The authors declare that they have no competing interests.

\section{Funding}

Ceva Santé Animal, France is the owner of Ceva Phylaxia SSIU and R\&D departments and funding all costs relevant to these studies. All authors are employed by Ceva Santé Animale, however allocated to and paid by different departments. This except the statistician Dr Nils Toft, these specific services paid by Ceva but accountable to own conclusions only.

\section{Authors' contributions}

PM generated this multi-analysis, gathered the data and acted as primary, corresponding author. NT analysed all data in the capacity of being a skilled independent statistician experienced in multi and meta- 
analyses. IK together with VP developed the Ceva aerosol chamber concept and are responsible for the production of the challenge data of the SSIU, plus reviewed and contributed importantly in writing. HS reviewed and contributed importantly in writing. MT is responsible for the production of the challenge data of the R\&D, plus reviewed and contributed importantly in writing.

\section{Acknowledgements}

Without whom nothing of this in reality would have happened: Márton Z, Szalai T, Pálmai N, Szórádi M-A, Baranyai B and Albert M, at Ceva-Phylaxia Bio R\&D, Felföldi B, and Kovács E, at Ceva-Phylaxia SSIU, and Halas M, and staff at the CRO, Prophyl Itd., Hungary, for their highly skilled, and dedicated performance in making all of this happen at the practical level, from taking care of bacterial cultures to pigs, and everything else imaginable, and sometimes non-imaginable in these several studies.

\section{References}

1. Bossé JT, Janson H, Sheehan BJ, Beddek AJ, Rycroft AN, Kroll JS, Langford PR. Actinobacillus pleuropneumoniae: pathobiology and pathogenesis of infection. Microbes Infect. 2002;4(2):225-35. doi: 10.1016/s1286-4579(01)01534-9. PMID: 11880056.

2. Opriessnig T, Giménez-Lirola LG, Halbur PG. Polymicrobial respiratory disease in pigs. Anim Health Res Rev. 2011;12(2):133-48. doi: 10.1017/S1466252311000120. PMID: 22152290.

3. Sassu EL, Bossé JT, Tobias TJ, Gottschalk M, Langford PR, Hennig-Pauka I. Update on Actinobacillus pleuropneumoniae-knowledge, gaps and challenges. Transbound Emerg Dis. 2018;65 Suppl 1:72-90. doi: 10.1111/tbed.12739. Epub 2017. PMID: 29083117.

4. Dreyfus A, Schaller A, Nivollet S, Segers RP, Kobisch M, Mieli L, Soerensen V, Hüssy D, Miserez R, Zimmermann W, Inderbitzin F, Frey J. Use of recombinant ApxIV in serodiagnosis of Actinobacillus pleuropneumoniae infections, development and prevalidation of the ApxIV ELISA. Vet Microbiol. 2004;99(3-4):227-38. https://doi.org 10.1016/j.vetmic.2004.01.004. PMID: 15066725.

5. Holmgren N, Lundeheim N, Wallgren P. Infections with Mycoplasma hyopneumoniae and Actinobacillus pleuropneumoniae in fattening pigs. Influence of piglet production systems and influence on production parameters. Zentralbl Veterinarmed B. 1999;46(8):535-44. doi: 10.1111/j.1439-0450.1999.tb01246.x. PMID: 10574070.

6. Gottschalk M, Broes A. Actinobacillosis. In: Zimmerman JJ, Karriker LA, Ramirez A, Schwartz KJ, Stevenson GW, Zhang J, eds Diseases of Swine, $11^{\text {th }}$ John Wiley \& Sons, Inc. 2019:749-66

7. Straw BE, Tuovinen VK, Bigras-Poulin M. Estimation of the cost of pneumonia in swine herds. J Am Vet Med Assoc. 1989;195(12):1702-6. PMID: 2689415.

8. Cárceles S, Cuestas F. Celma S, Oliver-Ferrando S, Del Carmen P, Carmona M, Lasierra M, Espigares D, Mortensen P. High Return on Investment Following Control of Actinobacillus Pleuropneumonia with an Actinobacillus Pleuropneumonia Vaccine Expression APX Toxins I, II and III under Field Conditions. Proc Asia Pig Vet Soc Con. 2019;9:148. 
9. Fraile L, Alegre A, López-Jiménez R, Nofrarías M, Segalés J. Risk factors associated with pleuritis and cranio-ventral pulmonary consolidation in slaughter-aged pigs. Vet J. 2010;184(3):326-33. doi: 10.1016/j.tvjl.2009.03.029. Epub 2009. PMID: 19527939.

10. Cleveland-Nielsen A, Nielsen EO, Ersbøll AK. Chronic pleuritis in Danish slaughter pig herds. Prev Vet Med. 2002;55(2):121-35. doi: 10.1016/s0167-5877(02)00089-2. PMID: 12350316.

11. Wellenberg GJ, Bouwkamp FT, Wolf PJ, Swart WA, Mombarg MJ, de Gee AL. A study on the severity and relevance of porcine circovirus type 2 infections in Dutch fattening pigs with respiratory diseases. Vet Microbiol. 2010;142(3-4):217-24. doi: 10.1016/j.vetmic.2009.10.003. Epub 2009. PMID: 19913367.

12. Jäger HC, McKinley TJ, Wood JL, Pearce GP, Williamson S, Strugnell B, Done S, Habernoll H, Palzer A, Tucker AW. Factors associated with pleurisy in pigs: a case-control analysis of slaughter pig data for England and Wales. PLoS One. 2012;7(2):e29655. doi: 10.1371/journal.pone.0029655. Epub 2012. PMID: 22363407; PMCID: PMC3281815.

13. Brewster VR, Maiti HC, Tucker AW, Nevel A. Associations between EP-like lesions and pleuritis and post trimming carcass weights of finishing pigs in England. Livestock Sci. 2017;201:1-4. ISSN 18711413, doi: 10.1016/j.livsci.2017.04.012.

14. Trachtman AR, Bergamini L, Palazzi A, Porrello A, Capobianco Dondona A, Del Negro E, Paolini A, Vignola G, Calderara S, Marruchella G. Scoring pleurisy in slaughtered pigs using convolutional neural networks. Vet Res. 2020;51(1):51. doi: 10.1186/s13567-020-00775-Z. PMID: 32276670; PMCID: PMC7149908.

15. Hannan PC, Bhogal BS, Fish JP. Tylosin tartrate and tiamutilin effects on experimental piglet pneumonia induced with pneumonic pig lung homogenate containing mycoplasmas, bacteria and viruses. Res Vet Sci. 1982;33(1):76-88. PMID: 7134653.

16. Merialdi G, Dottori M, Bonilauri P, Luppi A, Gozio S, Pozzi P, Spaggiari B, Martelli P. Survey of pleuritis and pulmonary lesions in pigs at abattoir with a focus on the extent of the condition and herd risk factors. Vet J. 2012;193(1):234-9. doi: 10.1016/j.tvjl.2011.11.009. Epub 2011. PMID: 22182431.

17. Sibila M, Aragón V, Fraile L, Segalés J. Comparison of four lung scoring systems for the assessment of the pathological outcomes derived from Actinobacillus pleuropneumoniae experimental infections. BMC Vet Res. 2014;10:165. doi: 10.1186/1746-6148-10-165. PMID: 25038822; PMCID: PMC4112831.

18. Jacobsen MJ, Nielsen JP, Nielsen R. Comparison of virulence of different Actinobacillus pleuropneumoniae serotypes and biotypes using an aerosol infection model. Vet Microbiol. 1996;49(3-4):159-68. doi: 10.1016/0378-1135(95)00184-0. PMID: 8734634.

19. Rosendal S, Miniats OP, Sinclair P. Protective efficacy of capsule extracts of Haemophilus pleuropneumoniae in pigs and mice. Vet Microbiol. 1986;12(3):229-40. doi: 10.1016/03781135(86)90052-0. PMID: 3776093.

20. Gerlach GF, Anderson C, Klashinsky S, Rossi-Campos A, Potter AA, Willson PJ. Molecular characterization of a protective outer membrane lipoprotein (OmIA) from Actinobacillus 
pleuropneumoniae serotype 1. Infect Immun. 1993;61(2):565-72. doi: 10.1128/iai.61.2.565-572.1993. PMID: 8423086; PMCID: PMC302765.

21. Furesz SE, Mallard BA, Bossé JT, Rosendal S, Wilkie BN, Maclnnes Jl. Antibody- and cell-mediated immune responses of Actinobacillus pleuropneumoniae-infected and bacterin-vaccinated pigs. Infect Immun. 1997;65(2):358-65. doi: 10.1128/iai.65.2.358-365.1997. PMID: 9009283; PMCID: PMC174603.

22. Bossé JT, MacInnes JI. Urease activity may contribute to the ability of Actinobacillus pleuropneumoniae to establish infection. Can J Vet Res. 2000;64(3):145-50. PMID: 10935879; PMCID: PMC1189605.

23. Tonpitak W, Baltes N, Hennig-Pauka I, Gerlach GF. Construction of an Actinobacillus pleuropneumoniae serotype 2 prototype live negative-marker vaccine. Infect Immun. 2002;70(12):7120-5. doi: 10.1128/IAI.70.12.7120-7125.2002. PMID: 12438394; PMCID: PMC133062.

24. Antenucci F, Fougeroux C, Deeney A, Ørskov C, Rycroft A, Holst PJ, Bojesen AM. In vivo testing of novel vaccine prototypes against Actinobacillus pleuropneumoniae. Vet Res. 2018;49(1):4. doi: 10.1186/s13567-017-0502-x. PMID: 29316978; PMCID: PMC5761136.

25. Bunka S, Christensen C, Potter AA, Willson PJ, Gerlach GF. Cloning and characterization of a protective outer membrane lipoprotein of Actinobacillus pleuropneumoniae serotype 5. Infect Immun. 1995;63(7):2797-800. doi: 10.1128/iai.63.7.2797-2800.1995. PMID: 7790104; PMCID: PMC173378.

26. Baltes N, Tonpitak W, Gerlach GF, Hennig-Pauka I, Hoffmann-Moujahid A, Ganter M, Rothkötter HJ. Actinobacillus pleuropneumoniae iron transport and urease activity: effects on bacterial virulence and host immune response. Infect Immun. 2001;69(1):472-8. doi: 10.1128/IAI.69.1.472-478.2001. PMID: 11119539; PMCID: PMC97905.

27. Maas A, Jacobsen ID, Meens J, Gerlach GF. Use of an Actinobacillus pleuropneumoniae multiple mutant as a vaccine that allows differentiation of vaccinated and infected animals. Infect Immun. 2006 Jul;74(7):4124-32. doi: 10.1128/IAl.00133-06. PMID: 16790786; PMCID: PMC1489739.

28. Buettner FF, Maas A, Gerlach GF. An Actinobacillus pleuropneumoniae arcA deletion mutant is attenuated and deficient in biofilm formation. Vet Microbiol. 2008;127(1-2):106-15. doi: 10.1016/j.vetmic.2007.08.005. Epub 2007. PMID: 17881160.

29. Buettner FF, Bendallah IM, Bosse JT, Dreckmann K, Nash JH, Langford PR, Gerlach GF. Analysis of the Actinobacillus pleuropneumoniae ArcA regulon identifies fumarate reductase as a determinant of virulence. Infect Immun. 2008;76(6):2284-95. doi: 10.1128/IAI.01540-07. PMID: 18378638; PMCID: PMC2423083.

30. Brauer C, Hennig-Pauka I, Hoeltig D, Buettner FF, Beyerbach M, Gasse H, Gerlach GF, Waldmann KH. Experimental Actinobacillus pleuropneumoniae challenge in swine: comparison of computed tomographic and radiographic findings during disease. BMC Vet Res. 2012;8:47. doi: 10.1186/17466148-8-47. PMID: 22546414; PMCID: PMC3537595.

31. Sebunya TN, Saunders JR, Osborne AD. Dose response relationship of Haemophilus pleuropneumoniae aerosols in pigs. Can J Comp Med. 1983;47(1):54-6. PMID: 6219732; PMCID: 
PMC1235884.

32. Sebunya TN, Saunders JR, Osborne AD. A model aerosol exposure system for induction of porcine Haemophilus pleuropneumonia. Can J Comp Med. 1983;47(1):48-53. PMID: 6403208; PMCID: PMC1235883.

33. Rosendal S, Maclnnes Jl. Characterization of an attenuated strain of Actinobacillus pleuropneumoniae, serotype 1. Am J Vet Res. 1990;51(5):711-7.

34. Brackmann J, Beckmann K, Lüken C, Baier S. Zur Verbreitung und Diagnostik von Actinobacillus pleuropneumoniae. Prakt Tierarzt. 2015;96(4):372-81. ISSN 0032-681 X.

35. Renken C. Seroprävalenz von Actinobacillus pleuropneumoniae sowie zugehöriger Serotypen und Vorkommen von Pleuritiden bei Mastschweinen aus Beständen mit klinischen Anzeichen einer Atemwegserkrankung. Inaugural-Dissertation zur Erlangung der Doktorwürde der Tierärztlichen Fakultät der Ludwig-Maximilians-Universität München 2017.

36. Jacques M, Labrie J, St Michael F, Cox AD, Paradis MA, Dick CP, Klopfenstein C, Broes A, Fittipaldi N, Gottschalk M. Isolation of an atypical strain of Actinobacillus pleuropneumoniae serotype 1 with a truncated lipopolysaccharide outer core and no 0-antigen. J Clin Microbiol. 2005;43(7):3522-5. doi: 10.1128/JCM.43.7.3522-3525.2005. PMID: 16000496; PMCID: PMC1169155.

37. Gottschalk M. The challenge of detecting herds sub-clinically infected with Actinobacillus pleuropneumoniae. Vet J. 2015;206(1):30-8. doi: 10.1016/j.tvjl.2015.06.016. Epub 2015 Jul 2. PMID: 26206322.

38. Gottschalk M, Lacouture S. Canada: Distribution of Streptococcus suis (from 2012 to 2014) and Actinobacillus pleuropneumoniae (from 2011 to 2014) serotypes isolated from diseased pigs. Can Vet J. 2015;56(10):1093-4. PMID: 26483588; PMCID: PMC4572831.

39. Min K, Chae C. Serotype and apx genotype profiles of Actinobacillus pleuropneumoniae field isolates in Korea. Vet Rec. 1999;145(9):251-4. doi: 10.1136/vr.145.9.251. PMID: 10504068.

40. Kim B, Hur J, Lee JY, Choi Y, Lee JH. Molecular serotyping and antimicrobial resistance profiles of Actinobacillus pleuropneumoniae isolated from pigs in South Korea. Vet Q. 2016;36(3):137-44. doi: 10.1080/01652176.2016.1155241. PMID: 26879953.

41. Stringer OW, Bossé JT, Lacouture S, Gottschalk M, Fodor L, Angen $\varnothing$, Velazquez E, Penny P, Lei L, Langford PR, Li Y. Proposal of Actinobacillus pleuropneumoniae serovar 19, and reformulation of previous multiplex PCRs for capsule-specific typing of all known serovars. Vet Microbiol. 2021;255:109021. doi: 10.1016/j.vetmic.2021.109021. PMID: 33667982.

42. Bossé JT, Li Y, Sárközi R, Fodor L, Lacouture S, Gottschalk M, Casas Amoribieta M, Angen $\varnothing$, Nedbalcova K, Holden MTG, Maskell DJ, Tucker AW, Wren BW, Rycroft AN, Langford PR; BRaDP1T consortium. Proposal of serovars 17 and 18 of Actinobacillus pleuropneumoniae based on serological and genotypic analysis. Vet Microbiol. 2018;217:1-6. doi: 10.1016/j.vetmic.2018.02.019. PMID: 29615241; PMCID: PMC5901230.

43. Renken C, Ritzmann M, Weiß C, Luppi A, Stoiber J, Waehner C, Eddicks M. Seroprevalence of Actinobacillus pleuropneumoniae and corresponding serotypes in fattening pigs in three different 
epidemiological regions in Germany. Proc. ESPHM/IPVS. 2016;PO-PF3-094:176

44. van Dixhoorn ID, Reimert I, Middelkoop J, Bolhuis JE, Wisselink HJ, Groot Koerkamp PW, Kemp B, Stockhofe-Zurwieden N. Enriched Housing Reduces Disease Susceptibility to Co-Infection with Porcine Reproductive and Respiratory Virus (PRRSV) and Actinobacillus pleuropneumoniae (A. pleuropneumoniae) in Young Pigs. PLoS One. 2016;11(9):e0161832. doi:

10.1371/journal.pone.0161832. PMID: 27606818; PMCID: PMC5015855.

45. Li L, Chen Z, Bei W, Su Z, Huang Q, Zhang L, Chen H, Zhou R. Catecholamines promote Actinobacillus pleuropneumoniae growth by regulating iron metabolism. PLoS One. 2015;10(4):e0121887. doi: 10.1371/journal.pone.0121887. PMID: 25849041; PMCID: PMC4388731.

46. Chiers K, De Waele T, Pasmans F, Ducatelle R, Haesebrouck F. Virulence factors of Actinobacillus pleuropneumoniae involved in colonization, persistence and induction of lesions in its porcine host. Vet Res. 2010;41(5):65. doi: 10.1051/vetres/2010037. PMID: 20546697; PMCID: PMC2899255.

47. Loera-Muro A, Angulo C. New trends in innovative vaccine development against Actinobacillus pleuropneumoniae. Vet Microbiol. 2018;217:66-75. doi: 10.1016/j.vetmic.2018.02.028. PMID: 29615259.

48. Frey J. Virulence in Actinobacillus pleuropneumoniae and RTX toxins. Trends Microbiol. 1995;3(7):257-61. doi: 10.1016/s0966-842x(00)88939-8. PMID: 7551637.

49. Frey J. RTX Toxins of Animal Pathogens and Their Role as Antigens in Vaccines and Diagnostics. Toxins (Basel). 2019;11(12):719. doi: 10.3390/toxins11120719. PMID: 31835534; PMCID: PMC6950323.

50. Fenwick BW, Osburn BI. Immune responses to the lipopolysaccharides and capsular polysaccharides of Haemophilus pleuropneumoniae in convalescent and immunized pigs. Infect Immun. 1986;54(2):575-82. doi: 10.1128/iai.54.2.575-582.1986. PMID: 3490442; PMCID: PMC260200.

51. Fenwick B, Henry S. Porcine pleuropneumonia. JAVMA. 1994;204:1334-40.

52. Ramjeet M, Deslandes V, St Michael F, Cox AD, Kobisch M, Gottschalk M, Jacques M. Truncation of the lipopolysaccharide outer core affects susceptibility to antimicrobial peptides and virulence of Actinobacillus pleuropneumoniae serotype 1. J Biol Chem. 2005;280(47):39104-14. doi: 10.1074/jbc.M502852200. PMID: 16188878.

53. Auger E, Deslandes V, Ramjeet M, Contreras I, Nash JH, Harel J, Gottschalk M, Olivier M, Jacques M. Host-pathogen interactions of Actinobacillus pleuropneumoniae with porcine lung and tracheal epithelial cells. Infect Immun. 2009;77(4):1426-41. doi: 10.1128/IAI.00297-08. PMID: 19139196; PMCID: PMC2663157.

54. Ramjeet M, Deslandes V, Gouré J, Jacques M. Actinobacillus pleuropneumoniae vaccines: from bacterins to new insights into vaccination strategies. Anim Health Res Rev. 2008;9(1):25-45. doi: 10.1017/S1466252307001338. PMID: 18346296.

55. Shao M, Wang Y, Wang C, Guo Y, Peng Y, Liu J, Li G, Liu H, Liu S. Evaluation of multicomponent recombinant vaccines against Actinobacillus pleuropneumoniae in mice. Acta Vet Scand. 2010;52(1):52. doi: 10.1186/1751-0147-52-52. PMID: 20831818; PMCID: PMC2944310. 
56. Thevenon J, Ivok M, Rozsnyay Z, Alapi I, Imre A, Tenk M. Coglapix, an Actinobacillus pleuropneumoniae inactivated vaccine induces high levels of anti-Apx and anti-capsular antibodies. Proc. ESPHM. 2014;P218:245

57. Tumamao JQ, Bowles RE, van den Bosch H, Klaasen HL, Fenwick BW, Storie GJ, Blackall PJ. Comparison of the efficacy of a subunit and a live streptomycin-dependent porcine pleuropneumonia vaccine. Aust Vet J. 2004;82(6):370-4. doi: 10.1111/j.1751-0813.2004.tb11108.x. PMID: 15272463.

58. van den Bosch $\mathrm{H}$, Frey J. Interference of outer membrane protein PalA with protective immunity against Actinobacillus pleuropneumoniae infections in vaccinated pigs. Vaccine. 2003;21(2526):3601-7. doi: 10.1016/s0264-410x(03)00410-9. PMID: 12922088.

59. European Pharmacopeia 10.0. Porcine actinobacillosis vaccine (inactivated). $2013 ; 10^{\text {th }}$ ED:1139-40.

60. Sárközi R, Makrai L, Fodor L. Actinobacillus pleuropneumoniae serotypes in Hungary. Acta Vet Hung. 2018;66(3):343-349. doi: 10.1556/004.2018.031. PMID: 30264610.

61. Bossé JT, Li Y, Fernandez Crespo R, Lacouture S, Gottschalk M, Sárközi R, Fodor L, Casas Amoribieta M, Angen $\varnothing$, Nedbalcova K, Holden MTG, Maskell DJ, Tucker AW, Wren BW, Rycroft AN, Langford PR; BRaDP1T consortium. Comparative sequence analysis of the capsular polysaccharide loci of Actinobacillus pleuropneumoniae serovars 1-18, and development of two multiplex PCRs for comprehensive capsule typing. Vet Microbiol. 2018;220:83-89. doi: 10.1016/j.vetmic.2018.05.011. PMID: 29885806; PMCID: PMC6008488.

62. Kleinsasser A, Olfert IM, Loeckinger A, Prisk GK, Hopkins SR, Wagner PD. Tidal volume dependency of gas exchange in bronchoconstricted pig lungs. J Appl Physiol (1985). 2007;103(1):148-55. doi: 10.1152/japplphysiol.00451.2006. PMID: 17395763.

63. Osborne AD, Saunders JR, Sebunya TK, Willson P, Green GH. A simple aerosol chamber for experimental reproduction of respiratory disease in pigs and other species. Can $\mathrm{J}$ Comp Med. 1985;49(4):434-5. PMID: 3935301; PMCID: PMC1236207.

64. Jones GF, Rapp-Gabrielson V, Wilke R, Thacker EL, Thacker BJ, Gergen L, Sweeney D, Washmoen T. Intradermal vaccination for Mycoplasma hyopneumoniae. J Swine Health Prod. 2005;13(1):19-27.

65. R Core Team (2019). R: A language and environment for statistical computing. R Foundation for Statistical Computing, Vienna, Austria. URL https://www.R-project.org/.

66. Bates D, Maechler M, Bolker B, Walker S. Fitting Linear Mixed-Effects Models Using Ime4. J Stat Softw. 2015;67(1):1-48. doi:10.18637/jss.v067.i01.

67. Kuznetsova A, Brockhoff PB, Christensen RHB (2017). ImerTest Package: Tests in Linear Mixed Effects Models. J Stat Softw. 2017;82(13):1-26. doi: 10.18637/jss.v082.i13 (URL: http://doi.org/10.18637/jss.v082.i13). 